BMJ Open Sport \& Exercise Medicine

\section{Clinical relevance of MRI knee abnormalities in Australian rules football players: a longitudinal study}

To cite: Aitken D, Balogun S, Foong YC, et al. Clinical relevance of MRI knee abnormalities in Australian rules football players: a longitudinal study. BMJ Open Sport \& Exercise Medicine 2021;7:e001097. doi:10.1136/ bmjsem-2021-001097

- Additional supplemental material is published online only. To view, please visit the journal online (http://dx.doi. org/10.1136/bmjsem-2021 001097)

$\mathrm{DA}$ and $\mathrm{SB}$ are joint first authors.

Accepted 30 August 2021

Check for updates

(C) Author(s) (or their employer(s)) 2021. Re-use permitted under CC BY-NC. No commercial re-use. See rights and permissions. Published by BMJ.

For numbered affiliations see end of article.

Correspondence to Dr Dawn Aitken; dawn.aitken@utas.edu.au

\section{ABSTRACT}

Background/Aim The clinical relevance of MRI knee abnormalities in athletes is unclear. This study aimed to determine the prevalence of MRI knee abnormalities in Australian Rules Football (ARF) players and describe their associations with pain, function, past and incident injury and surgery history.

Methods 75 male players (mean age 21, range 16-30) from the Tasmanian State Football League were examined early in the playing season (baseline). History of knee injury/surgery and knee pain and function were assessed. Players underwent MRI scans of both knees at baseline. Clinical measurements and MRI scans were repeated at the end of the season, and incident knee injuries during the season were recorded.

Results MRI knee abnormalities were common at baseline ( $67 \%$ bone marrow lesions, $16 \%$ meniscal tear/ extrusion, $43 \%$ cartilage defects, $67 \%$ effusion synovitis). Meniscal tears/extrusion and synovial fluid volume were positively associated with knee symptoms, but these associations were small in magnitude and did not persist after further accounting for injury history. Players with a history of injury were at a greater risk of having meniscal tears/extrusion, effusion synovitis and greater synovial fluid volume. In contrast, players with a history of surgery were at a greater risk of having cartilage defects and meniscal tears/extrusion. Incident injuries were significantly associated with worsening symptoms, BML development and incident meniscal damage.

Conclusions MRI abnormalities are common in ARF players, are linked to a previous knee injury and surgery history, as well as incident injury but do not dictate clinical symptomatology.

\section{INTRODUCTION}

Australian Rules Football (ARF) has a strong following in Australia, with over 1.4 million Australians participating in the sport. ${ }^{1}$ It has one of the highest injury rates among any sport played in Australia. ${ }^{23}$ Knee injuries are one of the most common and debilitating injuries sustained by ARF players, with significant short-term and long-term consequences. ${ }^{45}$ In the short term, players suffer from pain, loss

\section{Key messages}

What is already known

- MRI studies show that structural abnormalities are more common in athletes, but their clinical relevance is unclear.

What are the new findings

- This study found that MRI abnormalities were common in Australian Rules Football players, but that the relationship with symptoms was mediated by injury.

- This suggests no direct link between symptoms and MRI abnormalities.

- MRI abnormalities in athletes are unlikely to be clinically important in the absence of injury. If a player presents with an MRI abnormality but no clinical symptoms, further investigation or intervention is unlikely to be warranted.

of function and loss of playing time. ${ }^{6}$ In the long term, players are at an increased risk for developing osteoarthritis (OA) and future injury recurrence. ${ }^{67}$

MRI is a useful tool for diagnosing knee injuries and detecting early osteoarthritic abnormalities. MRI studies show that structural abnormalities are more common in athletes compared with matched controls. A higher proportion of both adolescent asymptomatic soccer players ${ }^{8}$ and elite swimmers ${ }^{9}$ had at least one knee MRI abnormality compared with controls $(64 \%$ and $69 \%$, respectively, compared with $32 \%$ of the control groups in each study). While studies consistently report that MRI changes resembling osteoarthritic changes are common in athletes, ${ }^{8-13}$ few studies ${ }^{14} 15$ have examined their clinical significance in terms of their relationship with pain, function and injury.

Determining the clinical significance of knee changes in athletes is important because early detection of harmful changes may help prevent OA development through better rehabilitation and targeted treatments. The aim of 
this study was to (1) determine the prevalence of knee abnormalities on MRI in a cohort of young ARF players, (2) describe the relationship of knee abnormalities with knee pain and past knee injury and surgery history, and (3) explore the relationship between incident knee injuries and change in knee structure throughout the season.

\section{METHODS}

\section{Participants}

This was a convenience sample of ARF players, and the data were collected twice over one playing season (2014) in Tasmania, Australia. Seventy-five male players (mean age 21, range 16-30 years) across four teams from the Tasmanian State Football League (top-level amateur league) were enrolled early in the season (baseline). Players had their height, weight and leg strength measured and provided questionnaire assessments of age, knee pain and function, and history of knee injuries and surgeries. Fifty-eight players underwent a baseline MRI scan of both knees. At the end of the season (mean follow-up $4.7( \pm 0.7)$ months), clinical measurements were repeated on 63, and MRI scans on 44 players. All participants provided written informed consent. Players, coaches and league officials were involved in the design of this study.

\section{Anthropometrics and leg strength}

Weight was measured using electronic scales (Heine, Dover, USA), and height was measured using the Leicester stadiometer (Invicta, Leicester, UK). Lower limb muscle strength was measured simultaneously for both limbs using a dynamometer (TTM Muscular Meter, Tokyo, Japan). Two trials were recorded, and the average of the two trials was taken as previously described.

\section{Knee pain and function}

Pain was assessed separately for the right and left knees using a $100 \mathrm{~mm}$ visual analogue scale (VAS) by asking, 'On this line, thinking about your RIGHT or LEFT knee, where would you rate your pain? Use the last seven days as a time frame'. A minimal clinically important difference (MCID) is $15 \mathrm{~mm}^{16}$

\section{Knee injury and surgery}

The knee injury and OA outcome score (KOOS) was also used to assess pain and function and has been validated in younger and athletic populations. ${ }^{17} 18$ Participants were asked 42 questions on 5 subscales: pain, symptoms, function/daily living, sport/recreation and quality of life (QOL). Each question had five response levels ranging from 0 to 4 (no pain/functional impairment to extreme pain/functional impairment). The MCID is 6, $5-8.5,7-8,5.8-12$ and $7-7.2$ points for the pain, symptoms, function/daily living, sport/recreation and QOL KOOS subscales respectively. ${ }^{19}$ A total KOOS score was calculated by summing the subscales, with 0 representing no knee problems and 168 representing extreme knee problems. An MCID value for the total KOOS score is not available.

History of knee injury/surgery was assessed using a selfadministered questionnaire. Players were asked whether they have ever had a knee injury/trauma or severe twisting of their RIGHT or LEFT knee and whether they have had previous knee surgery on their RIGHT or LEFT knee. Throughout the season, players kept an injury diary, providing information about incident knee injuries during the season.

\section{MRI}

MRI was acquired with a $1.5 \mathrm{~T}$ whole-body magnetic resonance unit (Siemens, Espree) using the following sequences: (1) a 2-dimensional proton density-weighted fat saturation fast spin echo acquisition sequence (2D-PDFS); (2) a 3-dimensional fat saturation Double Echo in the Steady State acquisition sequence (3D-DESS-FS); (3) an in/out phase T1-weighted gradient echo sequence, InOutGRE. The parameters are listed in online supplemental table 1 .

\section{Bone marrow lesions}

Bone marrow lesions (BMLs) were assessed on the 2D-PD-FS sequences and defined as areas of increased signal adjacent to the subcortical bone at the anterior and posterior medial tibial, medial femoral, lateral tibial, lateral femoral and patellar (superior and inferior) sites, by measuring the maximum area of the lesion $\left(\mathrm{mm}^{2}\right)$ as previously described.$^{20}$ This method has been shown to be more sensitive to change over time compared with an ordinal scoring system in a clinical trial. ${ }^{21}$ The intraclass correlation coefficient (ICC) was 0.97 for intraobserver repeatability. ${ }^{20}$ BML size at all sites was summed to create total BML size. Change in BML size was calculated as: end-of-season total BML size-early-season total BML size. Change in total BML size was analysed using the least significant criterion of $25 \mathrm{~mm}^{2}$ based on our previous work, indicating that only an increase larger than this represents a genuine change after considering observer variability in scoring BMLs. ${ }^{20}$ Figure 1 shows an example of an incident BML that developed during the season.

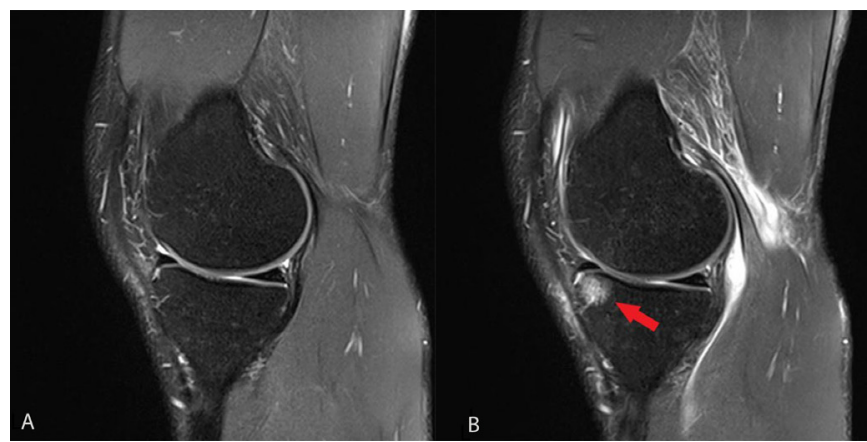

Figure 1 Example of an incident bone marrow lesion in a player that reported having a knee injury during the season. A tibial bone marrow lesion (red arrow) has developed between the early season (A) and the end of season (B). 


\section{Meniscal damage}

Meniscal damage was assessed on both the 3D-DESS-FS and 2D-PD-FS MR images and scored as previously described. $^{22}$ Meniscal tear and extrusion were scored separately at the anterior, middle, and posterior horns (medially/laterally). The intrareader and inter-reader ICC's range from 0.86 to 0.96 for meniscal tears and 0.85 to 0.92 for meniscal extrusions. ${ }^{23}$

\section{Cartilage defects}

Cartilage defects were scored using both the 3D-DESS-FS and 2D-PD-FS MR sequences at the medial tibial, medial femoral, lateral tibial, lateral femoral and patellar sites, as previously described ${ }^{22}$ from grade 0 (normal cartilage) to grade 4 (full-thickness chondral wear with exposure of subchondral bone). The presence of a cartilage defect was defined as a score of $\geq 2$. Incident cartilage defects were defined as a new cartilage defect in those with a score $<2$ at any site at baseline. ICCs ranged from 0.89 to 0.98 for intraobserver repeatability.

\section{Effusion synovitis}

Effusion synovitis was assessed as the presence of intraarticular fluid equivalent on the 2D-PD-FS sequences at the medial, central and lateral portions of the suprapatellar pouch. Effusion synovitis was scored according to the Whole-Organ MRI Score, graded from 0 to 3 in terms of the estimated maximal distention of the synovial cavity: 0 refers to normal; 1 to $<33 \%$ of maximum potential distention; 2 to $33 \%-66 \%$ of maximum potential distention; 3 to $>66 \%$ of maximum potential distention, as previously described. ${ }^{24}$ Pathological effusion synovitis was defined as any score of $\geq 2$. Incident effusion synovitis was defined as a new effusion synovitis in those with a score $<2$ at any site at baseline. The ICCs ranged from 0.71 to 0.88 for intraobserver repeatability.

\section{Synovial membrane thickness}

Synovial membrane thickness $(\mathrm{mm})$ was measured on the InOut-GRE sequence at four regions of interest (ROI) when synovial fluid was present in the corresponding ROI on the 2D-PD-FS: the medial and lateral suprapatellar bursa immediately above the patella and the medial and lateral recesses of the femur as previously described. ${ }^{25}$ The average of all available ROIs was used as a measure of synovial membrane thickness. The ICCs ranged from 0.89 to 0.99 for intraobserver repeatability.

\section{Synovial fluid volume}

Since the 3D-DESS-FS sequences offer an equivalent fluid contrast as a 2D-PD-FS with an enhanced in-image and in-slice resolution, synovial fluid volume $(\mathrm{mL})$ was measured on the 3D-DESS-FS images using a fully automated joint effusion volume quantification system as previously described. ${ }^{26}$

\section{Data analysis}

Hurdle models were used to describe the baseline associations between MRI abnormalities and knee pain and dysfunction. Log binomial models were used to (1) describe the baseline associations between a history of knee injury or surgery and MRI abnormalities and (2) describe the relationship between incident knee injuries during the season and changes in pain, function and MRI abnormality development/worsening. Correlation between observations on the same individual (right and left knee) was accounted for using clustered sandwich estimator with robust SE. Relative risk (RR) was reported for dichotomous outcomes and beta coefficients for continuous outcomes.

All models were adjusted for age, weight and height. Models examining the relationship between MRI abnormalities and knee pain and dysfunction were further adjusted for a history of a knee injury. Models examining the relationship between incident knee injuries and changes in pain and function were further adjusted for MRI abnormalities.

A $p$ value less than or equal to 0.05 (two tailed) was considered statistically significant. Statistical analyses were performed on Stata (V.16.0).

\section{RESULTS}

\section{Participant characteristics}

Table 1 presents the characteristics of participants. On average, the players played ARF for 11 years, 52\% reported having a previous knee injury, $9 \%$ previous knee surgery, and $76 \%$ of players reported having knee pain (defined as a VAS score $>0$ ) in at least one knee. Of 75,58 players had an MRI scan at baseline and 44 of 63 at follow-up. The primary reason for not having an MRI scan was accessibility and time restraints. Baseline characteristics of players who had an MRI scan $(n=58)$ were similar to those that did not $(\mathrm{n}=17)$, apart from a height difference $(185 \mathrm{~cm}$ vs $180 \mathrm{~cm}$ respectively, $\mathrm{p}<0.01)$.

\section{Prevalence of MRI knee abnormalities}

MRI knee abnormalities were common, with $67 \%$ of players having BMLs, $16 \%$ having meniscal tears or extrusions, $43 \%$ having cartilage defects and $67 \%$ having suprapatellar effusion-synovitis (table 1 ). Ninety per cent of players had at least one abnormality.

\section{MRI abnormalities and knee pain and function}

In multivariable analyses, meniscal tears/extrusion were associated with higher KOOS total, symptoms and stiffness scores after adjusting for age, weight and height. Still, the associations did not persist after further adjustment for history of knee injury (table 2). Synovial fluid volume was associated with higher KOOS total, symptoms, sport and QOL scores. After further adjustment for history of a knee injury, this relationship persisted only for QOL scores. BMLs were associated with lower KOOS sport scores but no other scores in the fully adjusted model.

\section{MRI abnormalities and previous injury or surgery}

Players with a history of injury were at a significantly greater risk of meniscal tears/extrusion and effusion 
Table 1 Characteristics of participants at baseline $(n=75)$

Mean (range) or $\%(n / N)$

\begin{tabular}{|c|c|}
\hline Age (years) & $21(16-30)$ \\
\hline Height (cm) & $183.6(170.6-202.3)$ \\
\hline Weight (kg) & $83.6(67.0-109.4)$ \\
\hline Years played AFL & $11(1-21)$ \\
\hline Leg strength (kg) & $187(25-280)$ \\
\hline Dominant kicking foot, right & $84 \%(53 / 63)$ \\
\hline Previous knee injury (right or left) & $52 \%(39 / 75)$ \\
\hline Right & $36 \%(27 / 75)$ \\
\hline Left & $34 \%(25 / 74)$ \\
\hline Previous knee surgery (right or left) & $9 \%(7 / 75)$ \\
\hline Right & $9 \%(7 / 75)$ \\
\hline Left & $3 \%(2 / 74)$ \\
\hline VAS pain*, (right and left) & $17(1-86)$ \\
\hline Right & $14(1-73)$ \\
\hline Left & $20(1-86)$ \\
\hline KOOS total ${ }^{*}$, (right and left) & $17(1-113)$ \\
\hline Right & $15(1-107)$ \\
\hline Left & $18(1-113)$ \\
\hline \multicolumn{2}{|l|}{ MRI prevalence data } \\
\hline Bone marrow lesions (right or left knee) & $67 \%(39 / 58)$ \\
\hline Right knee & $59 \%(34 / 58)$ \\
\hline Left knee & $47 \%(27 / 58)$ \\
\hline Meniscal tear (right or left knee) & $14 \%(8 / 58)$ \\
\hline Right knee & $12 \%(7 / 58)$ \\
\hline Left knee & $3 \%(2 / 58)$ \\
\hline Meniscal extrusion (right or left knee) & $2 \%(1 / 58)$ \\
\hline Right knee & $2 \%(1 / 58)$ \\
\hline Left knee & $0 \%(0 / 58)$ \\
\hline Cartilage defects (right or left knee)† & $43 \%(25 / 58)$ \\
\hline Right knee & $28 \%(16 / 58)$ \\
\hline Left knee & $33 \%(19 / 58)$ \\
\hline
\end{tabular}

Suprapatellar effusion synovitis (right or left $67 \%(39 / 58)$ knee) $\dagger$

\begin{tabular}{ll} 
Right knee & $52 \%(30 / 58)$ \\
Left knee & $50 \%(29 / 58)$ \\
\hline
\end{tabular}

*Summarised for those with VAS or KOOS scores $>0$.

†Defined as grade 2 or greater.

KOOS, Knee injury and osteoarthritis outcome score; $n$, total in sample; n, number with characteristic; VAS, visual analogue scale.

synovitis. They had greater synovial fluid volume after adjustment for age, weight and height (table 3). Players with a history of surgery were at significantly greater risk of having cartilage defects and meniscal tears/extrusion in the fully adjusted model.

\section{MRI abnormalities in asymptomatic players with no history of injury}

Of asymptomatic players (defined as a VAS score of 0 ) who had no history of injury or surgery $(n=20), 20 \%$ had
BMLs, $30 \%$ cartilage defects, $5 \%$ meniscal tear/extrusion and $50 \%$ effusion synovitis.

\section{Incident knee injuries during the season}

Ten players reported an incident knee injury. In multivariable analyses, players who reported having an incident knee injury had a significant increase in VAS score ( $\beta$ 29.6, 95\% CI 16.4 to 42.7 ), KOOS total score ( $\beta$ $26.5,95 \%$ CI 16.1 to 36.9 ), and in each KOOS subscale score apart from KOOS symptoms ( $\beta$ range 1.6 to 8.7 ) (table 4 ). The association between incident injury and symptoms was independent of MRI changes (data not shown). Players reporting incident injury also had a greater risk of developing a new or enlarging BML (RR $2.9,95 \%$ CI 1.5 to 5.9 ) and having incident meniscal damage (RR 4.0, 95\% CI 1.1 to 14.6). The effect size for the relationship between incident injury and incident cartilage defects was large but not statistically significant in multivariable analyses (RR 5.1, 95\% CI 0.4 to 75.4).

\section{DISCUSSION}

This study addresses an important evidence gap by determining the relationship between knee MRI abnormalities and clinical factors, including pain, function and history of injury and surgery in a cohort of top-level amateur Australian Rules Footballers. Despite MRI abnormalities being common, meniscal tears/extrusion and synovial fluid volume were the only abnormalities associated with symptoms, and a history of injury mediated these associations. Players who reported a previous knee injury were at a greater risk of having meniscal tears/extrusion, effusion synovitis and greater synovial fluid volume. In contrast, players reporting previous knee surgery were at a greater risk of having cartilage defects and meniscal tears/extrusion. Incident injuries were associated with worsening symptoms, independent of MRI changes. This suggests that the clinical relevance of knee MRI abnormalities in athletes varies and that they should be interpreted in the context of clinical presentations.

\section{Prevalence of MRI abnormalities in ARF players}

MRI abnormalities were common in this study, with the prevalence estimates being similar to that seen in other athletic populations. ${ }^{8911}$ Given that increased physical activity is associated with an increased risk of MRI abnormalities ${ }^{22}$ the high prevalence of MRI abnormalities in this cohort is not surprising. ARF is one of the most physically demanding and intense sports, with previous studies demonstrating that players in the elite league (AFL) cover an average of $13000 \mathrm{~m}$ per game with a high-intensity running distance of nearly $4000 \mathrm{~m} .{ }^{16}$ Understanding which MRI lesions lead to ongoing clinical symptoms or a higher risk of future knee OA is therefore of substantial importance.

\section{MRI abnormalities and pain and function}

While it is well established that MRI structural abnormalities are common in athletes, ${ }^{8-13}$ the clinical significance of 


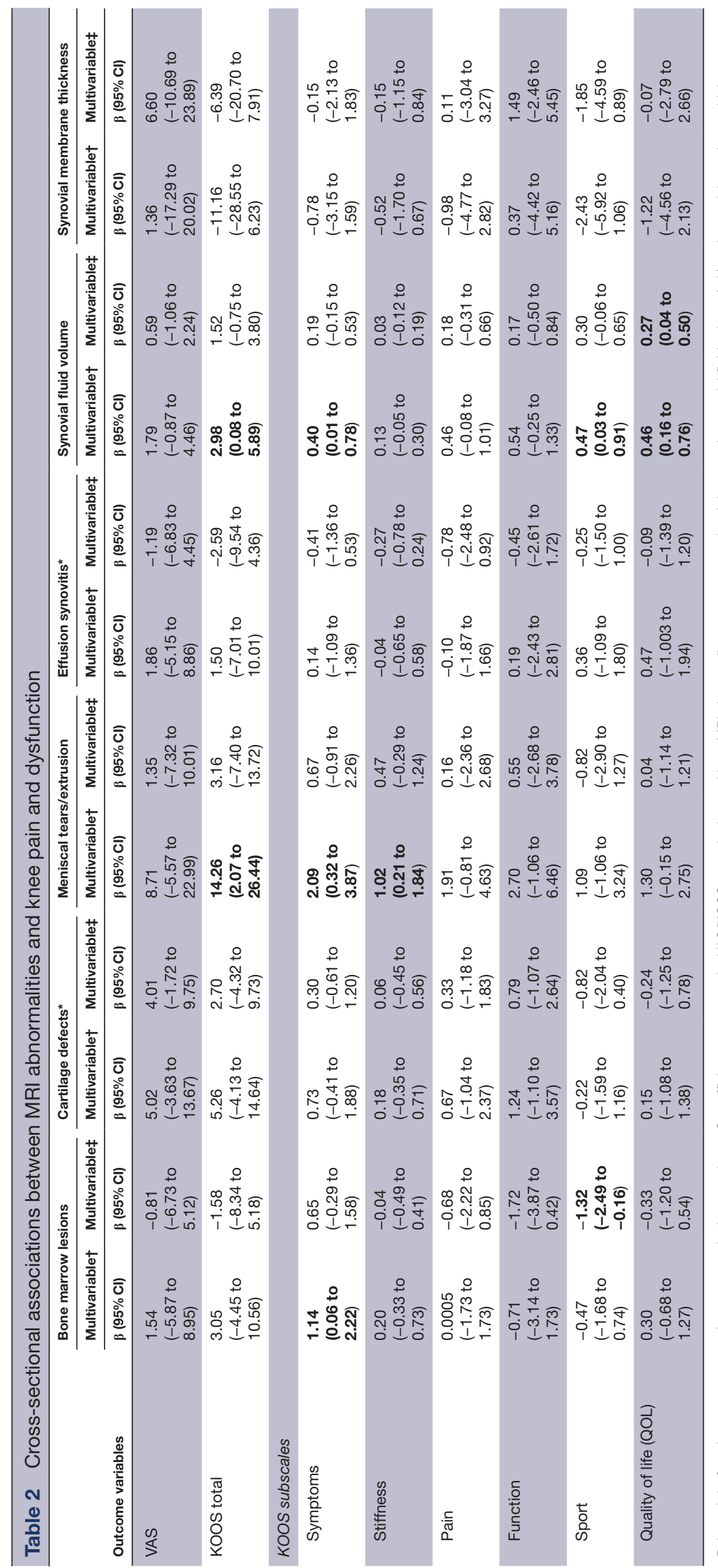


Table 3 Cross-sectional associations between a history of knee injury or surgery and MRI abnormalities

\begin{tabular}{|c|c|c|c|c|}
\hline \multirow[b]{3}{*}{ Outcome variables } & \multicolumn{2}{|l|}{ History of knee injury } & \multicolumn{2}{|l|}{ History of knee surgery } \\
\hline & Univariate & Multivariable $\dagger$ & Univariate & Multivariable $†$ \\
\hline & RR $(95 \% \mathrm{Cl})$ & RR $(95 \% \mathrm{Cl})$ & RR $(95 \% \mathrm{Cl})$ & RR $(95 \% \mathrm{Cl})$ \\
\hline BMLs & 1.41 (0.99 to 2.02$)$ & 1.30 (0.87 to 1.96$)$ & 1.49 (1.03 to 2.15$)$ & 1.29 (0.64 to 2.59$)$ \\
\hline Cartilage defects ${ }^{*}$ & 1.27 (0.75 to 2.17$)$ & 1.23 (0.70 to 2.17$)$ & 3.47 (2.28 to 5.27$)$ & $3.80(2.40$ to 6.01$)$ \\
\hline Meniscal tears/extrusion & 6.45 (1.57 to 26.62$)$ & 6.50 (1.59 to 26.49$)$ & 8.92 (3.41 to 23.32) & 14.25 (6.38 to 31.80$)$ \\
\hline \multirow[t]{2}{*}{ Effusion synovitis* } & 1.41 (0.98 to 2.01$)$ & 1.47 (1.03 to 2.09 ) & 1.54 (1.01 to 2.35$)$ & 1.68 (0.97 to 2.91$)$ \\
\hline & $\begin{array}{l}\text { Univariate } \\
\beta(95 \% \mathrm{Cl})\end{array}$ & $\begin{array}{l}\text { Multivariable† } \\
\beta(95 \% \mathrm{Cl})\end{array}$ & $\begin{array}{l}\text { Univariate } \\
\beta(95 \% \mathrm{Cl})\end{array}$ & $\begin{array}{l}\text { Multivariable† } \\
\beta(95 \% \mathrm{Cl})\end{array}$ \\
\hline Synovial fluid volume $(\mathrm{mL})$ & 1.25 (0.28 to 2.22$)$ & $1.02(0.17$ to 1.88$)$ & $2.50(0.27$ to 4.74$)$ & $1.85(-0.35$ to 4.05$)$ \\
\hline Synovial membrane thickness $(\mathrm{mm})$ & $-0.01(-0.09$ to 0.08$)$ & $-0.01(-0.10$ to 0.08$)$ & $0.04(-0.04$ to 0.12$)$ & $0.03(-0.06$ to 0.12$)$ \\
\hline
\end{tabular}

MRI abnormalities are the outcomes in these analyses.

RR represents the risk of having an MRI abnormality present in those who had a previous knee injury or surgery.

Boldface denotes statistically significant result.

$\beta$ coefficient represents the difference in synovial fluid volume $(\mathrm{mL})$ and synovial membrane thickness $(\mathrm{mm})$ in those who did and did not report a previous knee injury or surgery.

*Defined as grade 2 or higher.

†Adjusted for age, weight and height.

these abnormalities remains understudied. Interestingly, the only abnormalities associated with symptoms were meniscal tears/extrusion and synovial fluid volume, and these associations were very small in magnitude (based on the MCID's for the VAS $^{27}$ and $\operatorname{KOOS}^{19}$ subscales). They did not persist after further accounting for injury history. Injury history remained significant in these models (data not shown), demonstrating that its injury associated with symptoms (and not MRI abnormalities). We also found that MRI abnormalities can be present in the absence of knee pain or a history of knee injury/surgery. Altogether this suggests that in the absence of injury, MRI-detected abnormalities do not dictate clinical symptomatology. Surprisingly BMLs had an isolated association with lower

Table 4 The association between incident knee injuries $(n=10)$ and changes in pain, function and MRI abnormalities over the season

\begin{tabular}{|c|c|c|}
\hline \multirow[b]{3}{*}{ Outcome variables } & \multicolumn{2}{|c|}{ Incident knee injuries } \\
\hline & Univariable & Multivariable* \\
\hline & $\beta(95 \% \mathrm{Cl})$ & $\beta(95 \% \mathrm{Cl})$ \\
\hline Change in VAS & 27.53 (14.43 to 40.62$)$ & 29.57 (16.41 to 42.74$)$ \\
\hline Change in KOOS total & 25.97 (16.32 to 35.63$)$ & 26.48 (16.08 to 36.88$)$ \\
\hline \multicolumn{3}{|l|}{ KOOS subscales } \\
\hline Change in symptoms & $1.58(-0.27$ to 3.43$)$ & $1.68(-0.18$ to 3.54$)$ \\
\hline Change in stiffness & $1.55(0.84$ to 2.26$)$ & $1.61(0.89$ to 2.33$)$ \\
\hline Change in pain & 5.58 (3.46 to 7.70$)$ & 5.72 (3.45 to 7.98$)$ \\
\hline Change in function & $8.64(4.83$ to 12.46$)$ & $8.73(4.90$ to 12.56$)$ \\
\hline Change in sport & $4.65(2.41$ to 6.90$)$ & $4.69(2.47$ to 6.91$)$ \\
\hline Change in quality of life (QOL) & $3.96(2.61$ to 5.31$)$ & 4.05 (2.56 to 5.55$)$ \\
\hline Change in synovial membrane thickness & $0.12(-0.005$ to 0.25$)$ & $0.12(-0.02$ to 0.25$)$ \\
\hline \multirow[t]{3}{*}{ Change in synovial fluid volume } & $3.07(-1.39$ to 7.51$)$ & $3.34(-1.20$ to 7.87$)$ \\
\hline & Univariable & Multivariable* \\
\hline & RR $(95 \%$ Cl) & RR $(95 \% \mathrm{Cl})$ \\
\hline Development of a new or enlarging $\mathrm{BML} \dagger$ & $2.98(1.73$ to 5.14$)$ & $2.93(1.47$ to 5.87$)$ \\
\hline Incident cartilage defects $\ddagger$ & $6.33(1.04$ to 38.51$)$ & 5.12 (0.35 to 75.39$)$ \\
\hline Incident meniscal damage (tear, partial or full extrusion) & $4.84(1.37$ to 17.15$)$ & $3.96(1.07$ to 14.63$)$ \\
\hline Incident effusion synovitisł & $0.90(0.11$ to 7.49$)$ & $0.28(0.004$ to 19.25$)$ \\
\hline
\end{tabular}

Changes in pain, function and MRI abnormalities are the outcome in these analyses.

$\mathrm{RR}$ represents the risk of worsening MRI abnormalities in those who had an incident knee injury.

Boldface denotes statistically significant result.

$\beta$ coefficient represents the difference in change scores in those who did and did not report an incident knee injury.

${ }^{*}$ Adjusted for age, weight and height.

tDefined as an increase $>25 \mathrm{~mm}^{2}$ in total size.

$\neq$ Defined as a new cartilage defect/effusion synovitis in those with a score $<2$ at baseline

$\mathrm{BML}$, bone marrow lesion; KOOS, knee injury and osteoarthritis outcome score; VAS, visual analogue scale. 
KOOS sports scores only in the fully adjusted model. Still, the effect size was not clinically important and most likely represents a spurious finding.

Our study findings align with a recent study which concluded that MRI abnormalities (defined as MRIdefined $\mathrm{OA}$ ) were not associated with pain or function in a young athletic population. ${ }^{15}$ Interestingly and similarly to our study, they showed an association between injury history (reported 3-10 years previously) and the presence of MRI-defined OA. Still, they reported no direct link between MRI-defined OA and symptomology. ${ }^{15}$ Our findings are also in accordance with a case-control study that compared patients with patellofemoral pain to healthy controls and showed that the presence of MRI abnormalities of the patellofemoral joint was not associated with patellofemoral pain. ${ }^{14}$

\section{History of knee injury/surgery, incident knee injury and MRI abnormalities}

We found significant relationships between the history of injury or surgery with meniscal tears/extrusion, effusion synovitis, synovial fluid volume and cartilage defects. Furthermore, those players who reported an injury during the season were at a higher risk of BML development, incident meniscal damage and incident cartilage defects (although this was not statistically significant in the fully adjusted model) during the same season. These findings suggest that some MRI features in athletes may represent an acute response to or effect of injury, and that MRI changes post-injury/surgery can persist after the initial injury. ${ }^{28}{ }^{29}$ For example, Whittaker et al showed that young injured participants were 10 times more likely to have MRI abnormalities present $3-10$ years following their injury than uninjured participants. ${ }^{15}$

The relationship between a history of knee surgery and MRI abnormalities suggests that the severity of joint damage could lead to surgery. However, the reverse could be true, that surgery leads to abnormalities. Clarifying this relationship requires further longitudinal research in a cohort without a history of knee surgery.

\section{Implication for clinical practice}

Our study findings suggest that the clinical relevance of MRI abnormalities varies and that they should be interpreted in the context of clinical presentations. Athletes who present with MRI pathology following an injury may benefit from targeted OA prevention efforts. However, pathology detected on MRI may represent benign changes. Using MRI abnormalities alone to identify athletes who may benefit from early OA prevention interventions is not supported by current evidence. If a player presents with an MRI abnormality but no clinical symptoms, further investigation or intervention is unlikely to be warranted.

While MRI abnormalities in older adults and OA populations predict the development and progression of $\mathrm{OA}^{30}$ and are weakly to moderately associated with pain in these populations, ${ }^{31}$ the long-term impact of MRI abnormalities in athletes on joint health remains unclear.

\section{Strengths and limitations}

The strengths of this study include its longitudinal, cohort design, validated measures of pain, function and MRI abnormalities, and unique study population including both asymptomatic and symptomatic athletes with and without a history of a knee injury. There are also limitations. First, the participants are a small convenience sample recruited from the Tasmanian State Football League, limiting the generalisability of the findings, particularly to players of less elite levels of ARF. There was also attrition throughout the season. A larger study would be required to estimate the effects with greater precision so the effect estimates should be interpreted with caution. Second, the use of analgesics such as paracetamol or non-steroidal anti-inflammatory drugs, which could have been potential confounders, was not assessed.

\section{CONCLUSION}

MRI abnormalities are common in ARF players, are linked to a previous knee injury and surgery history, and incident injury, but do not dictate clinical symptomatology. This suggests that the clinical relevance of MRI abnormalities in ARF players varies, and their implication for longer term joint health needs further investigation.

\section{Author affiliations}

${ }^{1}$ Menzies Institute for Medical Research, University of Tasmania, Hobart, Tasmania, Australia

${ }^{2}$ National Centre for Epidemiology and Population Health, Australian National University, Canberra, Australian Capital Territory, Australia

${ }^{3}$ University Hospital Geelong, Geelong, Victoria, Australia

${ }^{4}$ The Sports Medicine Practice, Hobart, Tasmania, Australia

${ }^{5}$ School of Health Sciences, University of Tasmania, Hobart, Tasmania, Australia ${ }^{6}$ Osteoarthritis Research Unit, University of Montreal Hospital Centre Research Centre, Montreal, Québec, Canada

${ }^{7}$ Medical Imaging Research \& Development, ArthroLab Inc, Montreal, Québec, Canada

${ }^{8}$ Centre for Big Data Research in Health, University of New South Wales, Sydney, New South Wales, Australia

Twitter Dawn Aitken @DawnAitkenPhD

Acknowledgements We thank the players who made this study possible. We thank AFL Tasmania for supporting and promoting the study. We would like to acknowledge the clubs, Lauderdale, Hobart City Demons and Kingborough Tigers, for their support and assistance with recruitment. We also thank the study personnel, Kathy Buttigieg and Lizzy Reid for their role in data collection and Josée Thériault and André Pelletier for their reading of the MR images.

Contributors All authors were involved in drafting the article or revising it for important intellectual content. All authors have approved the final manuscript. DA ( dawn.aitken@utas.edu.au) takes responsibility for the integrity of the work as a whole, from inception to finished article. Conception and design: DA, DH, LL, NP, FA, JP-P, JMP, GJ, TW. Data collection: DA, LL, NP, HK, XJ. Data management and cleaning: DA, YCF, SB, HK, FA, J-PP, JM-P, XJ. Analysis and interpretation of data: all authors.

Funding DA, LL, XJ and GJ are recipients of National Health and Medical Research Council of Australia (NHMRC) fellowship funding to support their salaries.

Competing interests J-PP and JM-P are shareholders in ArthroLab. FA is an employee of ArthroLab.

Patient and public involvement Patients and/or the public were involved in the design, or conduct, or reporting, or dissemination plans of this research. Refer to the Methods section for further details. 
Patient consent for publication Not applicable.

Ethics approval This study was approved by the Tasmanian Health and Medical Human Research Ethics Committee (H0013667).

Provenance and peer review Not commissioned; externally peer reviewed.

Data availability statement Data are available upon reasonable request. The data generated from this study will not be deposited in a public repository due to privacy and consent restrictions. Deidentified data can be made available from the corresponding author on reasonable request, subject to a data sharing agreement.

Open access This is an open access article distributed in accordance with the Creative Commons Attribution Non Commercial (CC BY-NC 4.0) license, which permits others to distribute, remix, adapt, build upon this work non-commercially, and license their derivative works on different terms, provided the original work is properly cited, appropriate credit is given, any changes made indicated, and the use is non-commercial. See: http://creativecommons.org/licenses/by-nc/4.0/.

ORCID iD

Dawn Aitken http://orcid.org/0000-0001-5685-7634

\section{REFERENCES}

1 Australian Football League. Australian football League annual report. Melbourne, Victoria, Australia, 2016.

2 Ekegren CL, Gabbe BJ, Donaldson A, et al. Injuries in communitylevel Australian football: results from a club-based injury surveillance system. J Sci Med Sport 2015;18:651-5.

3 Finch C, Da Costa A, Stevenson M, et al. Sports injury experiences from the Western Australian sports injury cohort study. Aust N Z J Public Health 2002;26:462-7.

4 Hrysomallis C. Injury incidence, risk factors and prevention in Australian rules football. Sports Med 2013;43:339-54.

5 Orchard J, Seward H. Epidemiology of injuries in the Australian football League, seasons 1997-2000. Br J Sports Med 2002;36:39-44.

6 Warren P, Gabbe BJ, Schneider-Kolsky M, et al. Clinical predictors of time to return to competition and of recurrence following hamstring strain in elite Australian footballers. Br J Sports Med 2010;44:415-9.

7 Deacon A, Bennell K, Kiss ZS, et al. Osteoarthritis of the knee in retired, elite Australian rules footballers. Med J Aust 1997;166:187-90

8 Soder RB, Simões JD, Soder JB, et al. MRI of the knee joint in asymptomatic adolescent soccer players: a controlled study. AJR Am J Roentgenol 2011;196:W61-5.

9 Soder RB, Mizerkowski MD, Petkowicz R, et al. MRI of the knee in asymptomatic adolescent swimmers: a controlled study. Br J Sports Med 2012;46:268-72.

10 Boeth $\mathrm{H}$, MacMahon A, Eckstein F, et al. MRI findings of knee abnormalities in adolescent and adult volleyball players. J Exp Orthop 2017;4:6.

11 Kornaat PR, de Jonge MC, Maas M. Bone marrow edema-like signa in the athlete. Eur J Radiol 2008:67:49-53.

12 Kornaat PR, Van de Velde SK. Bone marrow edema lesions in the professional runner. Am J Sports Med 2014;42:1242-6.

13 Pappas GP, Vogelsong MA, Staroswiecki E, et al. Magnetic resonance imaging of asymptomatic knees in collegiate Basketball players: the effect of one season of play. Clin J Sport Med 2016;26:483-9.

14 van der Heijden RA, de Kanter JLM, Bierma-Zeinstra SMA, et al. Structural abnormalities on magnetic resonance imaging in patients with Patellofemoral pain: a cross-sectional case-control study. Am J Sports Med 2016;44:2339-46.

15 Whittaker JL, Toomey CM, Woodhouse LJ, et al. Association between MRI-defined osteoarthritis, pain, function and strength
3-10 years following knee joint injury in youth sport. Br J Sports Med 2018;52:934-9.

16 Coutts AJ, Quinn J, Hocking J, et al. Match running performance in elite Australian rules football. J Sci Med Sport 2010;13:543-8.

17 Cameron KL, Thompson BS, Peck KY, et al. Normative values for the KOOS and WOMAC in a young athletic population: history of knee ligament injury is associated with lower scores. Am J Sports Med 2013;41:582-9.

18 Salavati M, Akhbari B, Mohammadi F, et al. Knee injury and osteoarthritis outcome score (KOOS); reliability and validity in competitive athletes after anterior cruciate ligament reconstruction. Osteoarthritis Cartilage 2011:19:406-10.

19 Collins NJ, Misra D, Felson DT, et al. Measures of knee function: international knee documentation Committee (IKDC) subjective knee evaluation form, knee injury and osteoarthritis outcome score (KOOS), knee injury and osteoarthritis outcome score physical function short form (KOOS-PS), knee outcome survey activities of daily living scale (KOS-ADL), Lysholm knee scoring scale, Oxford knee score (OKS), Western Ontario and McMaster universities osteoarthritis index (WOMAC), activity rating scale (ARS), and Tegner activity score (Tas). Arthritis Care Res 2011;63 Suppl 11:S208-28.

20 Doré D, Quinn S, Ding C, et al. Natural history and clinical significance of MRI-detected bone marrow lesions at the knee: a prospective study in community dwelling older adults. Arthritis Res Ther 2010;12:R223.

21 Laslett LL, Doré DA, Quinn SJ, et al. Zoledronic acid reduces knee pain and bone marrow lesions over 1 year: a randomised controlled trial. Ann Rheum Dis 2012;71:1322-8.

22 Doré DA, Winzenberg TM, Ding C, et al. The association between objectively measured physical activity and knee structural change using MRI. Ann Rheum Dis 2013;72:1170-5.

23 Raynauld J-P, Martel-Pelletier J, Berthiaume M-J, et al. Long term evaluation of disease progression through the quantitative magnetic resonance imaging of symptomatic knee osteoarthritis patients: correlation with clinical symptoms and radiographic changes. Arthritis Res Ther 2006:8:R21.

24 Wang X, Blizzard L, Halliday A, et al. Association between MRIdetected knee joint regional effusion-synovitis and structural changes in older adults: a cohort study. Ann Rheum Dis 2016;75:519-25.

25 Pelletier J-P, Raynauld J-P, Abram F, et al. A new non-invasive method to assess synovitis severity in relation to symptoms and cartilage volume loss in knee osteoarthritis patients using MRI. Osteoarthritis Cartilage 2008;16 Suppl 3:S8-13.

26 Li W, Abram F, Pelletier J-P, et al. Fully automated system for the quantification of human osteoarthritic knee joint effusion volume using magnetic resonance imaging. Arthritis Res Ther 2010;12:R173.

27 Tubach F, Ravaud P, Martin-Mola E, et al. Minimum clinically important improvement and patient acceptable symptom state in pain and function in rheumatoid arthritis, ankylosing spondylitis, chronic back pain, hand osteoarthritis, and hip and knee osteoarthritis: results from a prospective multinational study. Arthritis Care Res 2012;64:1699-707.

28 Nepple JJ, Wright RW, Matava MJ, et al. Full-thickness knee articular cartilage defects in national football League combine athletes undergoing magnetic resonance imaging: prevalence, location, and association with previous surgery. Arthroscopy 2012;28:798-806.

29 Potter HG, Jain SK, Ma Y, et al. Cartilage injury after acute, isolated anterior cruciate ligament tear: immediate and longitudinal effect with clinical/MRI follow-up. Am J Sports Med 2012;40:276-85.

30 Hayashi D, Guermazi A, Kwoh CK. Clinical and translational potential of MRI evaluation in knee osteoarthritis. Curr Rheumatol Rep 2014:16:391.

31 Yusuf E, Kortekaas MC, Watt I, et al. Do knee abnormalities visualised on MRI explain knee pain in knee osteoarthritis? A systematic review. Ann Rheum Dis 2011;70:60-7. 